\title{
Investigation of an Optically Induced Superstrate Plasma for Tuning Microstrip Antennas
}

\author{
C. D. Gamlath ${ }^{1}$, M.A. Collett ${ }^{1}$, W. Pang ${ }^{1}$, D.M. Benton ${ }^{2}$ and M. J. Cryan ${ }^{* 1}$ \\ ${ }^{1}$ Department of Electrical and Electronic Engineering, University of Bristol, Bristol, BS81TR, \\ U.K. \\ ${ }^{2}$ Aston Institute of Photonics Technologies, Aston University, Aston Triangle, Birmingham, \\ B47ET, U.K. \\ *m.cryan@bristol.ac.uk
}

\begin{abstract}
Optically induced electron-hole plasmas in silicon are used to perform radiation pattern tuning. The antenna is a slot loaded microstrip patch and the effect of illumination is shown to produce beam switching in the radiation patterns of certain modes while other modes are left unaffected. The structure is specifically designed to make the best use of currently available miniature laser sources to form a compact tunable package. Modelled and measured results for tuning of the radiation patterns and frequency response are presented. The effect of the losses incurred by the plasma along with the losses in the optically transparent ground plane are quantified in both simulation and measurement. This forms the basis for designing other types of optically tunable miniature antennas based on the structure presented.
\end{abstract}

\section{Introduction}

In today's crowded mobile environment the ability of an antenna to dynamically alter its beam pattern is a highly desirable feature. This allows the antenna to selectively receive from different transmitters at different times depending on the tuning state chosen. The recent push towards higher frequency bands for use in communication networks means that tuning technologies will need to operate at the higher end of the microwave spectrum. Conventional tuning technologies are typically based on PIN and varactors diodes [1] or more recently MEMS switches [2]. While these approaches work well at low microwave frequencies, complex bias networks in large tunable arrays are difficult to implement. At higher frequencies (> 30GHz) such bias networks begin to dominate the array performance making these tuning approaches very difficult. This paper looks at the viability of using optical tuning methods where no bias lines are required which thus offers a very scalable approach to large array tuning.

Microwave tuning based on optical illumination of semiconductors has been investigated for many years. The basis for this technique is the creation of an optically illuminated plasma through the application of illumination on semiconductor materials [3]. One very important feature of a semiconductor plasma is that since there is no PN junction present very linear behaviour can be obtained [4]. There can be residual 
Schottky contacts formed at metal-semiconductor interfaces, but with careful control of metal type and process these can be made very ohmic. Finally the use of optical switching may allow very high speed switching to be obtained and switching speeds down to picoseconds have been observed [5].The properties of the plasma remain consistent with increasing frequency and this tuning method can be used from the lower end of the microwave region up to mmWave frequencies [6]. The losses in the plasma and the extra power required for the pump laser are the two disadvantages of this technique. However the continuing developments in vertical-cavity surface-emitting laser(VCSEL) arrays and micro light emitting diode array technologies mean that the efficiency of light sources is improving steadily thus decreasing the DC power requirement [7] while maintaining a small form factor.

Previous investigations on optically tunable antennas have looked at the possibility of creating the entire antenna out of optically illuminated patches $[8,9]$. We have recently investigated this approach as well [10]. However such methods require large amounts of optical power and are suitable mostly for pulsed applications. The use of high power lasers also means that small form factors are not possible. Other investigations have focused on the use of optically controlled switches mounted on various parts of the antenna [11,12]. While these structures work well at the lower range of microwave frequencies, the parasitics of the switch start to become problematic at higher frequencies.

The tuning method described here investigates the effect of integrating the silicon layer as part of the antenna's superstrate. This has several advantages, firstly due to the high permittivity of silicon, the incorporation of this layer increases the gain of the antenna. Previous works have shown that high losses are incurred when the diffusion tail of the plasma extends into the substrate. By forming the plasma on the superstrate, the effect of the diffusion tail is inverted thus minimizing the losses incurred. It also allows any optically transparent material such as air or fused silica to be used as the substrate layer. By choosing a low dielectric substrates the antenna efficiency can be improved. Since the silicon superstrate is part of the antenna structure the need for mounting switches is eliminated hence the operating frequency could be extended beyond the microwave band.

A double slotted microstrip patch antenna was chosen as the candidate architecture. The illumination area inside the slot could be made small enough such that the power requirement was kept to a minimum thus allowing miniature laser sources to be used. These could then be integrated into the antenna package thus forming a compact tunable antenna structure. Although the final integration is not performed here as it required precision structures to be fabricated, these are well within the manufacturing capabilities of standard industrial processes. The basis for operation of slotted microstrip patch antennas $[13,14,15]$ and the effect of superstrates [16] on slots have been investigated in the past. The theory of operation for the antenna 
presented here combines these two concepts to form a tunable slot loaded antenna. The effect of illumination alters the behaviour of the antenna from a slotted patch to that of an unslotted patch as the conductivity of the plasma covers the slot. The nulls in the radiation pattern due to the presence of the slots are thus altered as shown in the later sections.

Section 2 describes the construction of the antenna and section 3 describes the basis for the tuning method. Section 4 describes the modelling methods used and also shows the simulation results using a full wave solver. The measurements showing the varying radiation patterns of the constructed antenna are then presented in section 5 .

\section{Antenna Construction and Layout}

The antenna investigated was a rectangular microstrip antenna with two slots close to the radiating edges as shown in Fig.1a. This structure was chosen for several reasons. The structure has been well studied in the past and the changes of the nulls in the beam pattern of the antenna from the slotted to the unslotted state has been shown to create significant variations in the beam patterns of certain antenna modes. Only certain modes were strongly affected by the presence of the slots hence the effect of illumination on the unaffected modes could also be investigated through the same structure. The illumination area could be made small enough such that the power requirement was well within the capabilities of miniature laser sources.

An optically transparent substrate is used to feed the illumination from the underside, allowing for complete integration in future systems. The ground plane is formed out of a thin layer of Indium Tin Oxide (ITO) obtained from Nancos Inc. ITO is an optically transparent but electrically conductive material, this forms an optically transparent ground plane through which the laser illumination can pass. A 975nm VCSEL array (Princeton Optronics 975nm C-Mount) is used as the illumination source. The illumination wavelength of $975 \mathrm{~nm}$ was used due to the high carrier generation efficiency in silicon at this wavelength. A schematic of the structure is shown in Fig.1b. An optical cage system was used for the illumination optics. In order to maximize the amount of power that was collected from the VCSEL the collimating lens was required to have a short focal length with high Numerical Aperture. The Thorlabs C390TME-B was found to be the best option. Since the focal length had to be kept short the beam would start to diverge almost immediately after the plane of the lens.

Some of this divergence was corrected using the homogenizing rod light pipe (Edmund Optics NT63092) with a $25 \mathrm{~mm}$ length and a hexagonal cross-section of $2 \mathrm{~mm}$. The illumination setup shown in Fig. $1 \mathrm{~b}$ was for experimental purposes only as it eliminates the need for a microlens array. For practical applications 
the VCSEL array can be mounted directly below the $100 \mu \mathrm{m}$ glass layer and the entire optical system replaced by a microlens array thus allowing for a compact structure.

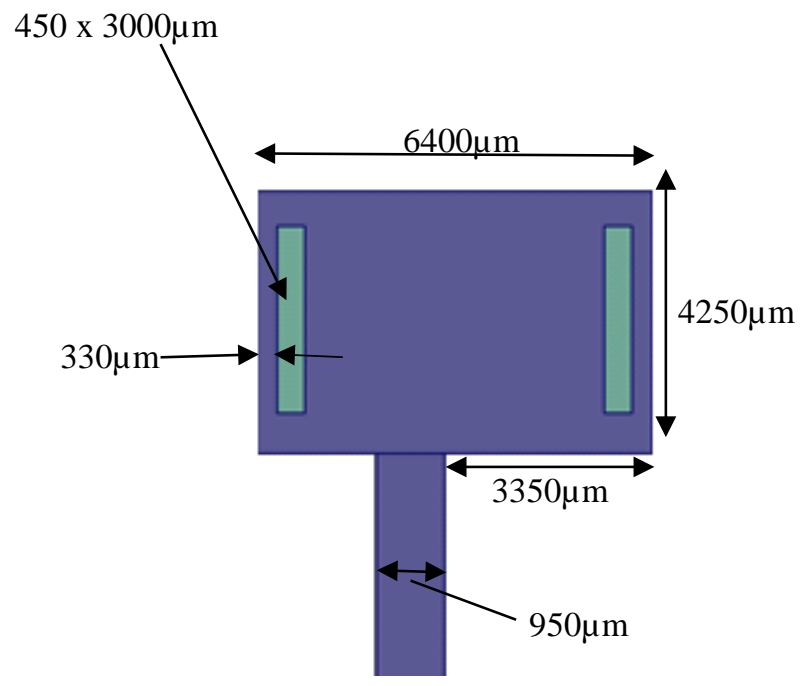

(a)

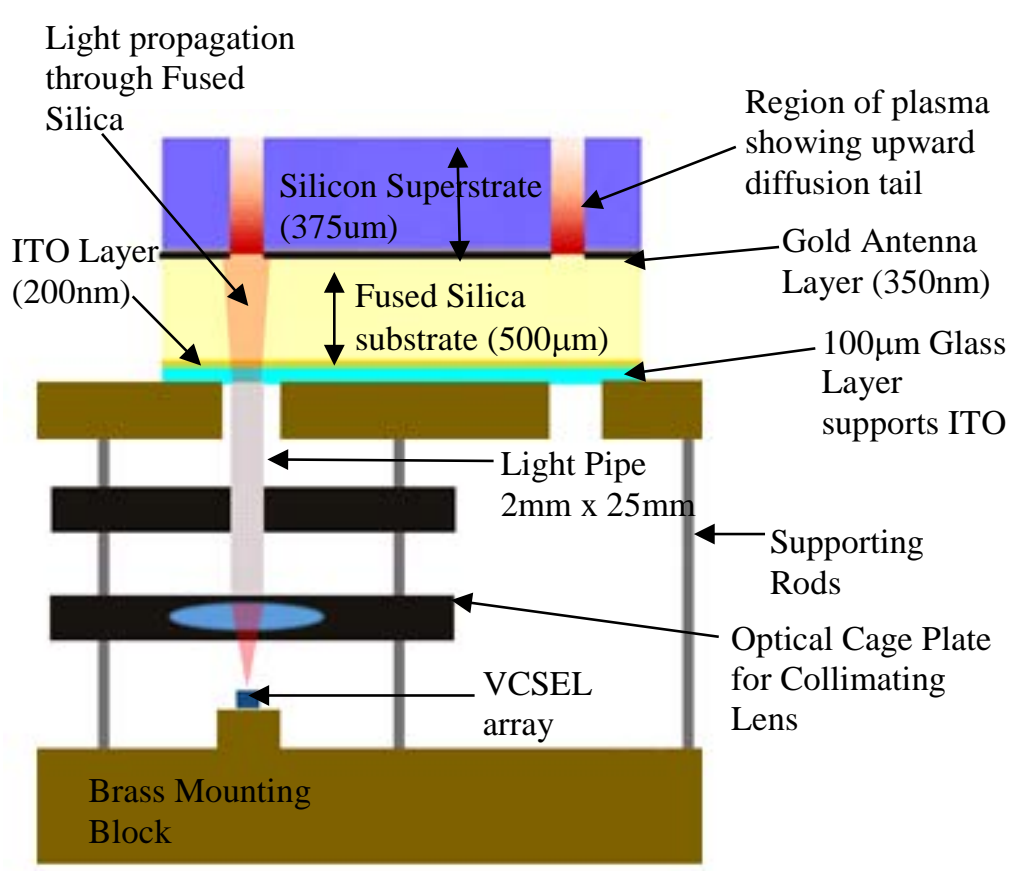

(b)

Fig. 1 (a) Top view of proposed antenna structure (b) Side view of antenna structure showing layer stack showing optical system used to illuminate one slot. 
The transparent low permittivity $(\varepsilon r=3.3)$ and low loss of the fused silica substrate also increased the efficiency of the antenna. The high permittivity silicon layer which forms the superstrate could be used to increase the gain by increasing directivity. Since the illumination is from underneath and is unaffected by the superstrate thickness, this can be further optimized to increase directivity for the desired angle of radiation [17]. Multi-layered superstrates can also be used as long as the first layer is kept as the optically illuminated semiconductor layer. The antenna constructed here has not been optimized for any particular angle, thus the thickness of the superstrate was kept low at $375 \mu \mathrm{m}$. The structure also takes advantage of the fact that the maximum of the conductivity profile appears just above the metal layer. This minimizes losses incurred in the plasma as explained in the modelling section (section 4).

The basis for the tuning mechanism is explained in the next section followed by modelling methods and the simulation results.

\section{THEORY OF OPERATION}

The effect of slots on rectangular microstrip patch antennas has been investigated extensively $[13,14,15]$. The presence of the slot perturbs the current profile of the resonant modes in the patch. By illuminating the slot region and hence creating the plasma in the slot, the currents around the slot can be altered depending on the intensity of the plasma and this forms the basis for tuning.

The simulated results of $S_{11}$ for the resonant modes with and without the plasma applied is shown in Fig. 2. Also shown is the $S_{11}$ for an unslotted antenna which is equivalent to covering the slots with metal. Since the effect of the presence of the plasma is similar to covering the slots with metal, this case shows how closely the plasma approximates a metal covering. The individual mode numbers are labelled on Fig. 2 and follow the standard $\mathrm{TM}_{\mathrm{z}}$ naming convention for microstrip antennas where the $\mathrm{z}$ direction points into the depth of the substrate. Comparison of the graphs show that while most of the resonant modes have some frequency shift, the $\mathrm{TM}_{200}$ mode is the most strongly perturbed. 


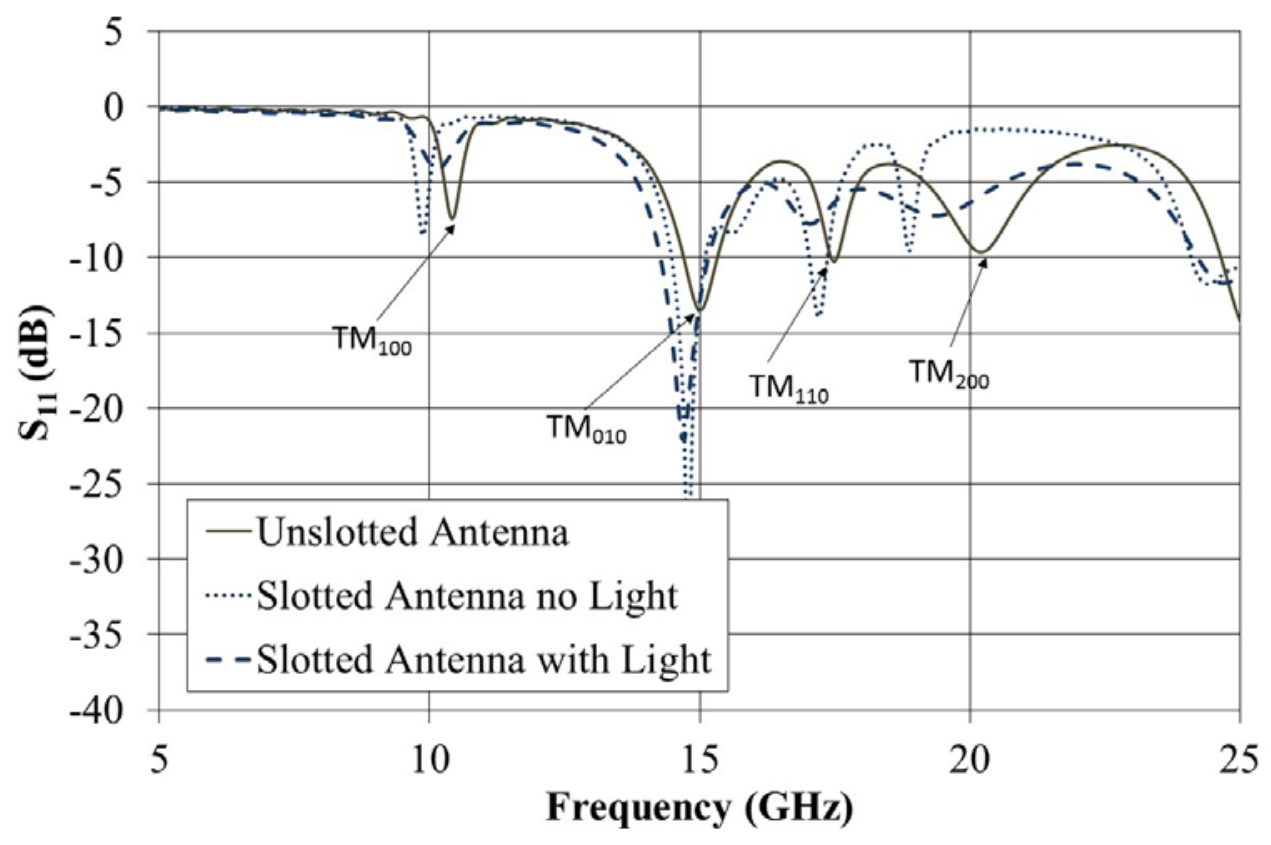

Fig. 2. Simulated $S_{11}$ results for patch antenna with and without illumination.

The reason for this is that this mode has strong currents close to the edges of the slots as shown in Fig. 3a which are strongly perturbed with the application of the plasma. Hence a mode switching effect is achieved as shown in Fig. 3b. A detailed theoretical analysis of mode switching for slot loaded microstrip antennas requires the calculation of equivalent circuit models for modelling the effect of the slots as described in [18]. A detailed circuit analysis of the plasma loaded patch is beyond the scope of this paper but previous works form a good basis for this analysis [19]. The advantage of tuning the individual resonant modes is that some modes can be perturbed while the other modes are unaffected by the presence of the plasma. This provides advantages for the design of multiband tunable antennas.

The overall effect of illuminating the slot is to shift the antenna behaviour closer to the unslotted state as can be seen in Fig. 2. However due to the finite conductivity and phase shift caused by the plasma the results show some differences. This is particularly apparent around the $\mathrm{TM}_{200}$ higher order mode.

Although the $\mathrm{TM}_{010}$ and $\mathrm{TM}_{110}$ modes do not show much frequency shift in $\mathrm{S}_{11}$, the effect of the plasma does alter the surface currents of these modes causing changes to nulls of the radiation pattern as shown later. The losses incurred in the plasma are shown in the next section. 


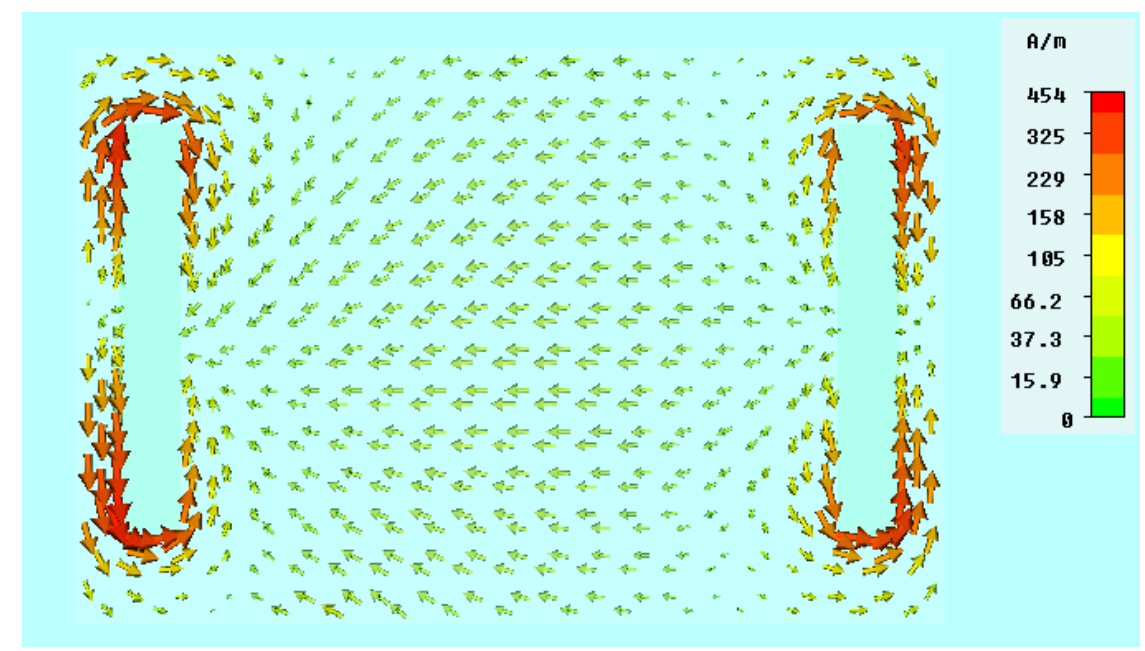

(a)

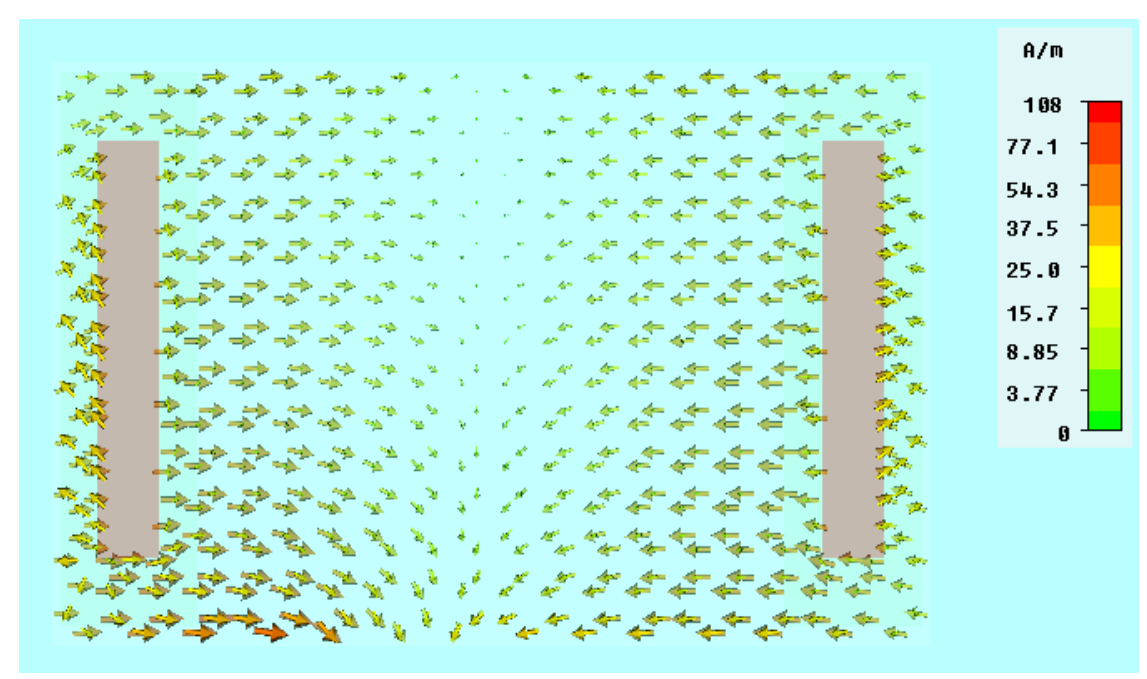

(b)

Fig. 3 (a) Surface currents for the $T M_{200}$ mode at 20.17Ghz (a) without illumination (b) with illumination.

\section{MODELLING OF LOSSES}

When a region of semiconductor is illuminated an electron hole plasma is formed at the surface [20]. The diffusion mechanism within the semiconductor causes the conductivity profile of the plasma to spread exponentially through the depth of the material. This can cause significant losses if a significant portion of the E-field exists in the region of low conductivity. 
Fig. 4 shows the plasma applied to a single slot of the antenna. Unlike the case shown in [20], the region of maximum conductivity is now in line with the region of maximum field strength. The E-field in the space between the slot decays rapidly into the depth of the silicon so that the lower layers of plasma interact very little with the E-field. This causes the lower layers of the plasma having low conductivity to contribute very little to the overall losses. The surface conductivity of the plasma then becomes the most dominant factor for the loss.

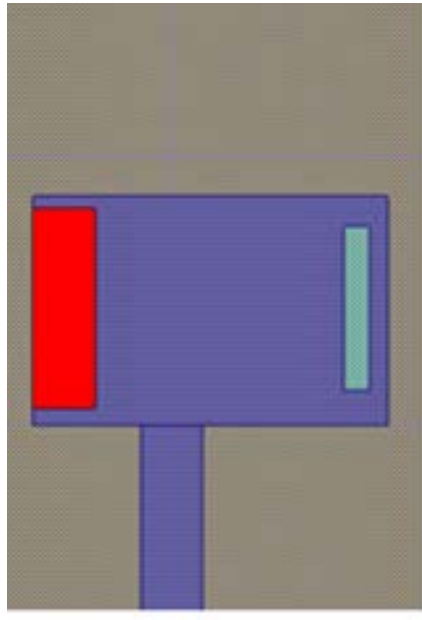

(a)

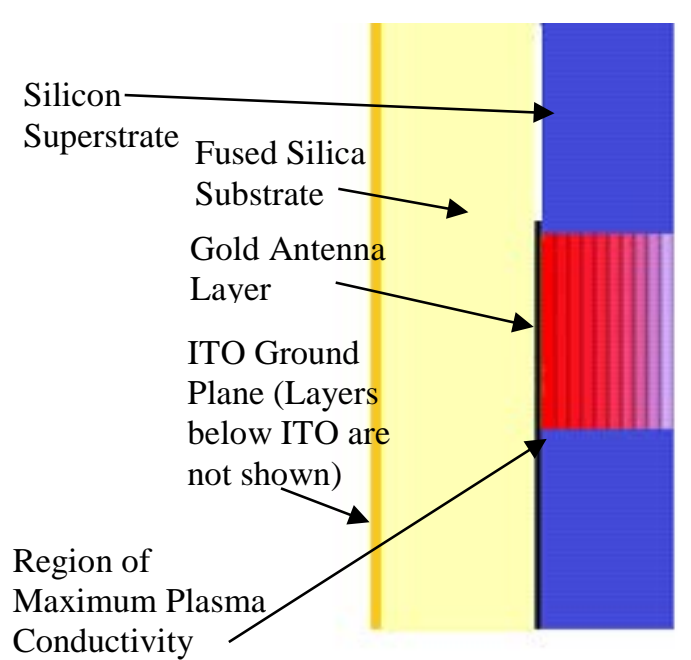

(b)

Fig. 4. (a)Top view of antenna with illumination applied to one slot and (b) Magnified side view of plasma region showing plasma layers .

A second source of loss in the antenna was due to the thickness of the ITO ground plane being less than the skin depth. The skin depth of ITO for around $20 \mathrm{GHz}$ was calculated to be $5 \mu \mathrm{m}$ but the physical thickness of the ITO layer was only 200nm. Since the ITO layer has a near metal conductivity in the region of $5 \times 10^{5} \mathrm{~S} / \mathrm{m}$, the modelling of the ITO layer could be done using an equivalent zero thickness layer of lossy metal. This was found to greatly reduce the simulation time instead of the direct inclusion of a metal layer of 200nm thickness. The basis for this equivalence is described below.

The standard model for the surface impedance of a metal assumes that the thickness of the metal is much greater than the skin depth at the metal surface. The metal surface can then be modelled as an infinitesimally thin layer having an impedance of equal value to the surface impedance of the metal. However in our case, since the ITO thickness is smaller than the skin depth of ITO, the surface impedance required modification to account for this reduction in thickness. This lead to a modified value of the conductivity which was much lower than the actual value. The modified surface impedance, $Z_{s}$ is shown in Eq. 1 [21].

$$
Z_{s}=\frac{k}{\sigma} \frac{e^{k t}+e^{-k t}}{e^{k t}-e^{-k t}}
$$

Where $\sigma$ is the conductivity of ITO, $t$ is the thickness of the ITO layer and $k$ is given by Eq 2 . 


$$
k=\frac{1+j}{\delta}
$$

Where $\delta$ is the skin depth of the ITO which can be easily calculated using the standard equation for skin depth [22]. Eq.1 shows that the surface impedance is composed of both real and imaginary parts. For a conductor with a thickness less than the skin depth, the real part dominates the impedance and the imaginary part can hence be ignored. The ITO layer is thus modelled as a resistive sheet with a lower conductivity value. Using the values of $\sigma=5.5 \times 10^{5} \mathrm{~S} / \mathrm{m}, \mathrm{t}=200 \mathrm{~nm}$ and $\omega=2 \pi f$ where $f=20 \mathrm{GHz}$ shows that the conductivity of needs to be decreased to around $1 \times 10^{3} \mathrm{~S} / \mathrm{m}$ in order to account for its lower thickness. This was used in the simulations and showed good agreement with the measurements shown in Fig. 7b of the next section. Further details of the method can be found in [21].

The equivalent conductivity calculated above was for the case when the entire ground plane was formed out of ITO. Using a similar process it can be shown [21] that the equivalent conductivity is increased by placing a thick metal layer immediately behind the ITO layer. Referring back to Fig. 1b it is seen that the ITO layer sits on top of a 100um insulating glass layer which is required for supporting the ITO. If this layer was inverted the ITO layer would sit immediately next to the thick brass layer. Thus according to the above discussion this would increase the overall conductivity of the ground plane. This is found to be the case in the measured results shown in section 5 where the inverted layer structure is compared to that in Fig. $1 \mathrm{~b}$. The measured results for the radiation pattern were taken with the layer structure identical to that of Fig. $1 \mathrm{~b}$. Since the radiation patterns were nearly the same with the inverted layer structure only a comparison of gain is shown in Fig. 10c.

The changes to the radiation patterns with the application of illumination is shown in the following section.

\section{MEASUREMENTS}

Pattern measurements for the unilluminated antenna were carried out in an anechoic chamber. Fig. 5 shows the measured directivity of the unilluminated antenna along with the simulated results which show reasonable agreement. In order to get accurate comparisons for the changes to the nulls with the illumination applied, the measurements had to be taken with the antenna position fixed and the illumination being turned on and off at each angle. 


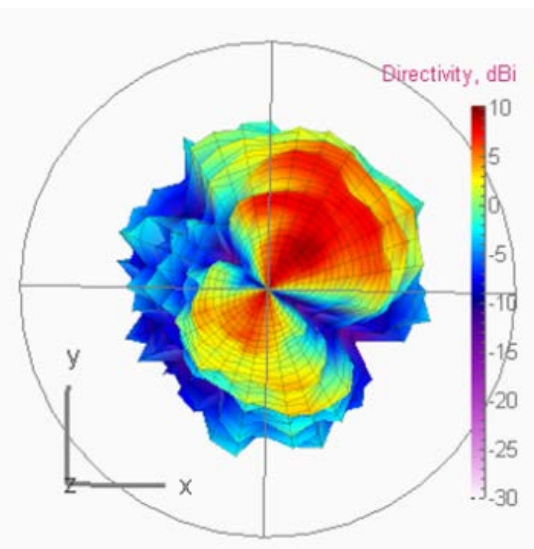

(a)

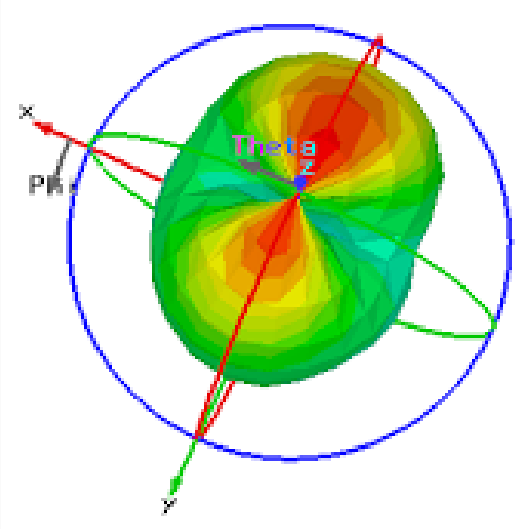

(b)

Fig. 5. (a) Measured 3D radiation pattern of unilluminated antenna in anechoic chamber. (b) Simulated pattern for the TM100 at $10 \mathrm{GHz}$.

This was not possible in the anechoic chamber setup which had to complete one full cycle before the illumination state could be changed. The measurements in the chamber also had a frequency limit of $12 \mathrm{GHz}$ due to the cables inside the chamber. Hence it was necessary to build a custom pattern measurement setup as shown in Fig. 6. The E-Plane and H-Plane results for the unilluminated antenna were compared with the anechoic chamber measurement setup and showed good agreement. 


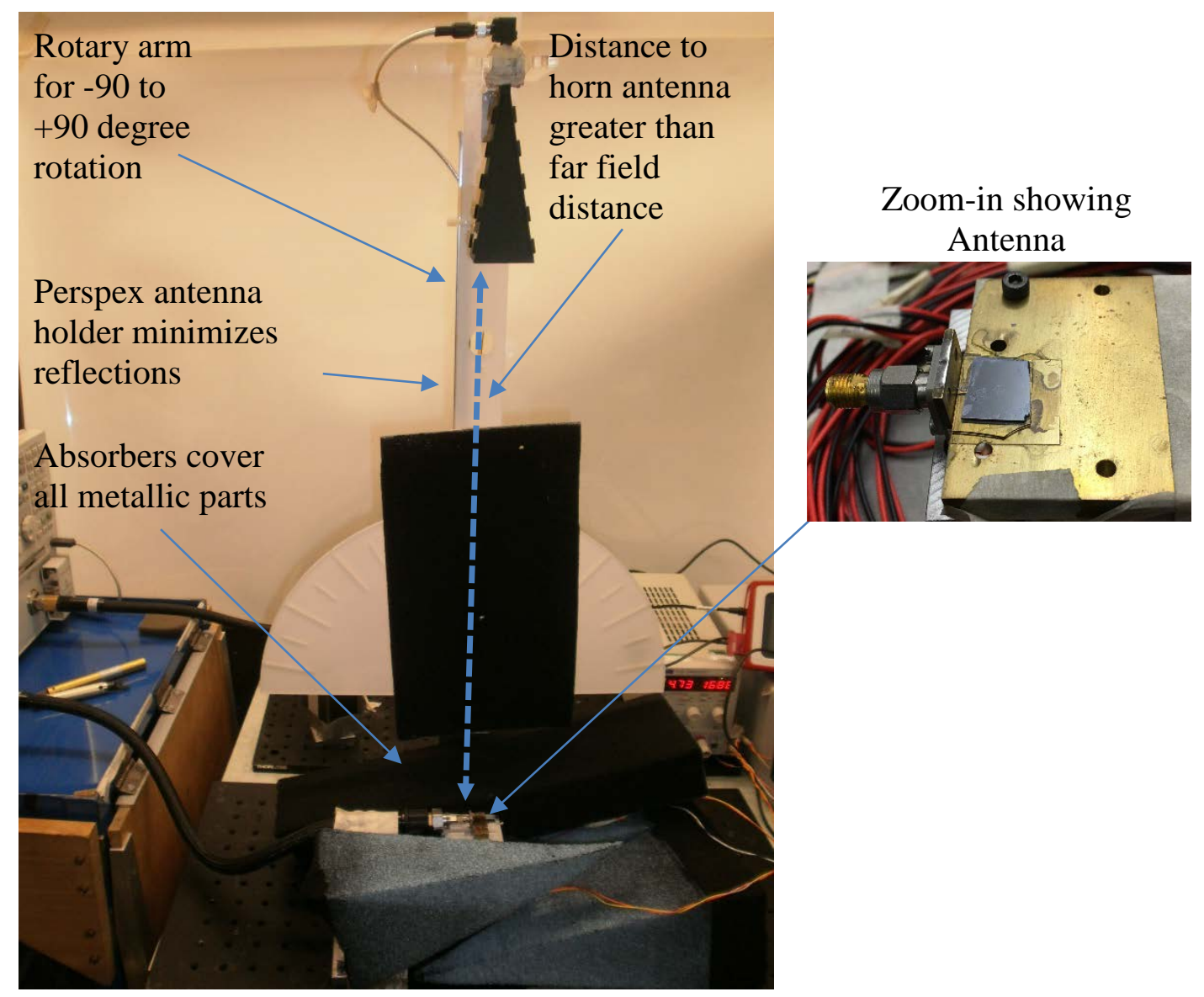

Fig. 6. Custom measurement setup for antenna radiation patterns and zoom-in showing details of antenna, superstrate silicon can be seen which is mounted on top of the antenna.

The $\mathrm{S}_{11}$ return loss measurements for the unilluminated antenna were compared with the simulations as shown in Fig. 7a. This showed good agreement with the simulated frequency of each mode. The results with the ITO ground plane added are shown in Fig. 7b. The effect of using the equivalent conductivity of the ITO sheet as described in the modelling section shows a closer match to the measured results as shown in this figure. This can be clearly seen around $15 \mathrm{GHz}$ where the modified value of ITO conductivity shows a closer match to the measurements. In some cases the resonant dips are masked by the resistance. The TM 010 and $\mathrm{TM}_{110}$ modes remain clearly visible but the $\mathrm{TM}_{100}$ and $\mathrm{TM}_{200}$ mode are seen to disappear almost completely.

For the case of the $\mathrm{TM}_{200}$ mode this is also due to the feed position of the constructed antenna being not well matched with this mode. Due to the nature of the field profiles for the three modes it was not possible to find a single feed position that would perfectly match all four modes simultaneously. However the changes to the radiation pattern could still be measured for these modes and this feeding method was sufficient for investigation purposes. 


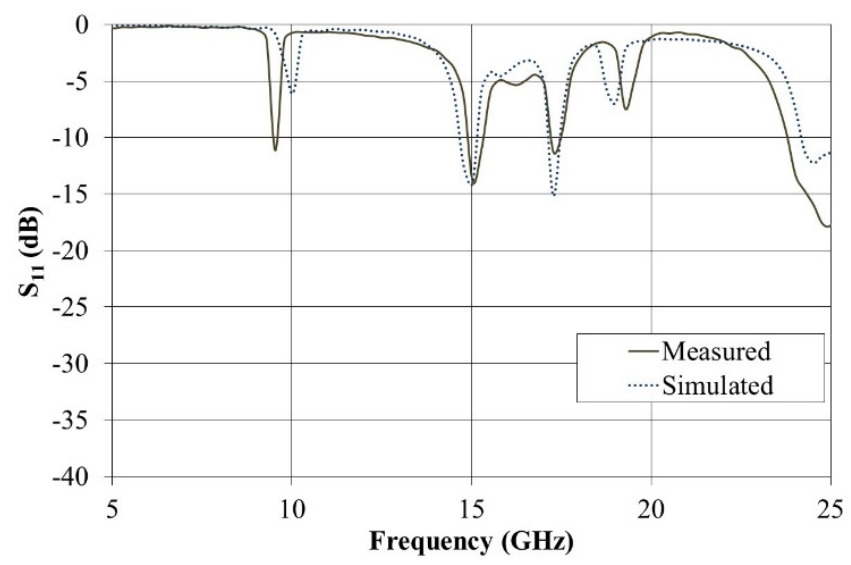

(a)

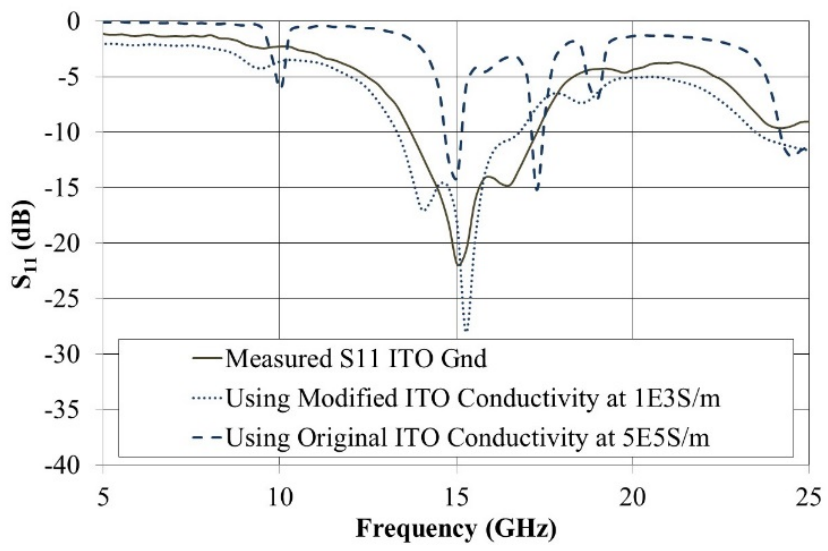

(b)

Fig. 7 Simulated and measured $S_{11}$ with (a) Copper ground plane. (b) ITO ground plane.

The changes in radiation pattern due to illumination were measured for each of the four modes. The results were taken using the setup in Fig. 6 with illumination applied to the left slot only as shown in Fig. 4. The orientation of the measurement was chosen such that the change in the nulls was highlighted for each mode. Fig. 8a shows the measured $\mathrm{TM}_{100}$ mode under the illuminated and unilluminated states. Fig. 8b shows the corresponding cut through the simulation result with reasonable agreement with the measured results. There is no significant effect on the radiation pattern in this case aside from a minor decrease in gain due to the addition of extra losses in the plasma. Figs. 8c and 8d shows the measured and modelled results for the $\mathrm{TM}_{110}$ mode. Here the illumination causes a radiation null to appear while leaving the rest of the main lobe radiation nearly unchanged. The modelled results show a similar change in the null feature at a slightly different angle, most likely due to the difficultly in replicating the measured radiation environment in this type of set up. 


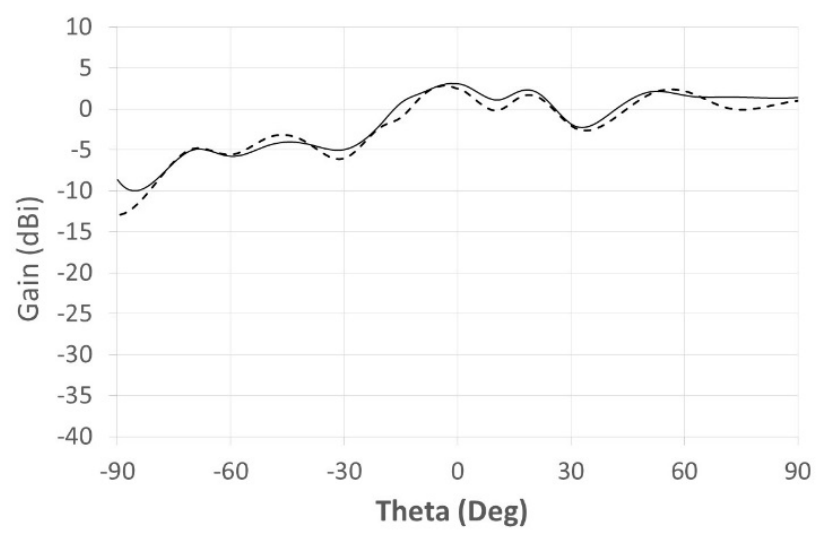

(a)

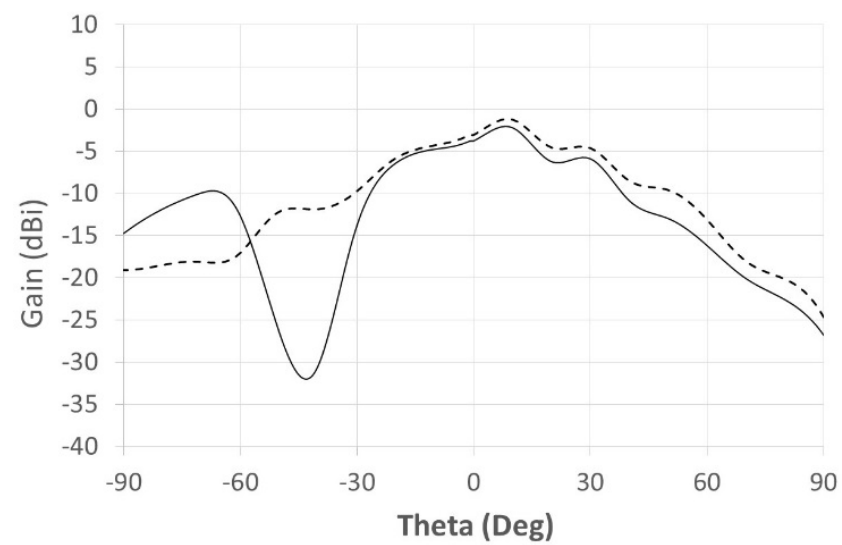

(c)

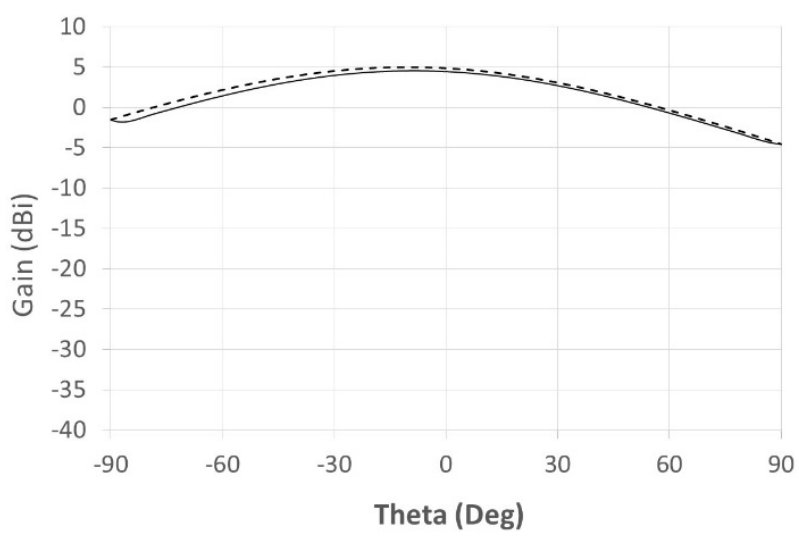

(b)

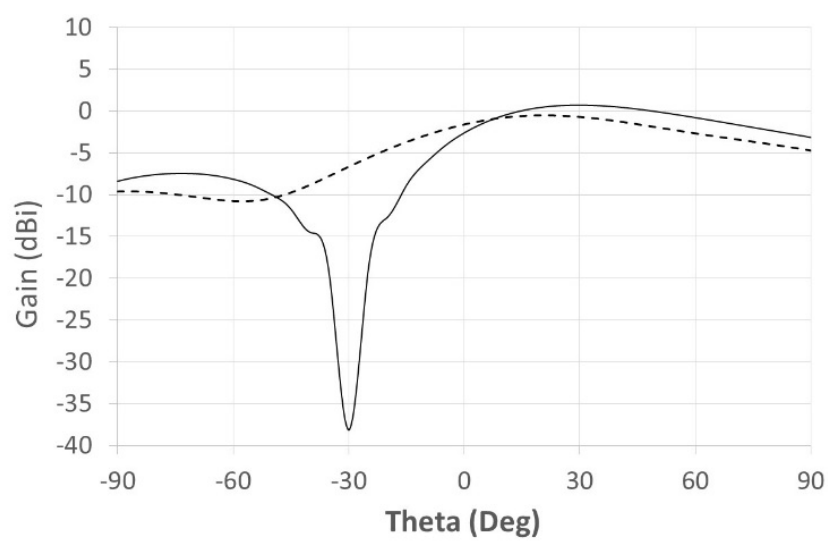

(d)

Fig. 8. (a) Measured and (b) Simulated results for TM100 mode at 10GHz, (c) Measured and (d) Simulated Results for $T M_{110}$ at $17 \mathrm{GHz}$, dashed : without illumination, solid : with illumination

Figs. 9a and 9b show the effect of illumination for the $\mathrm{TM}_{010}$ mode. Here the radiation null is removed by the illumination which causes an increase in gain of $20 \mathrm{~dB}$ and similar effects is observed in the modelled results. Figs. 9c and 9d show the radiation pattern changes for the $\mathrm{TM}_{200}$ mode and they show that the radiation null improves by $20 \mathrm{~dB}$ with again reasonable agreement with modelled results.

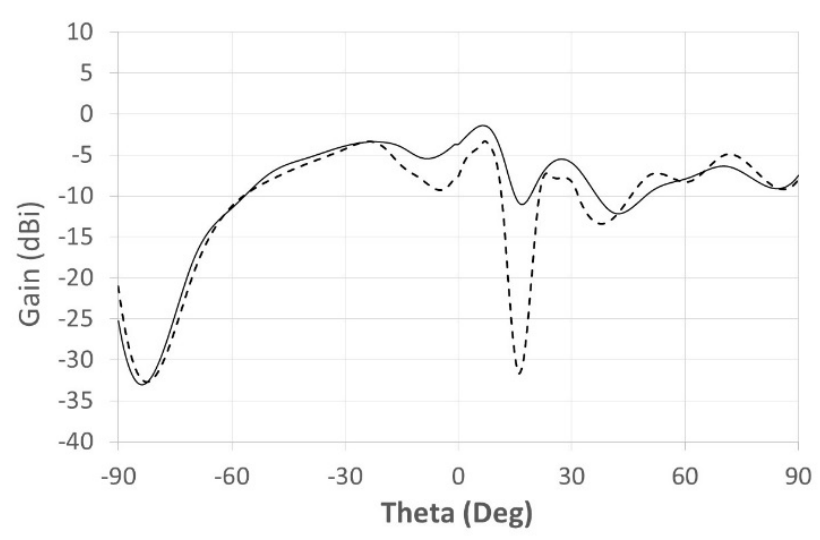

(a)

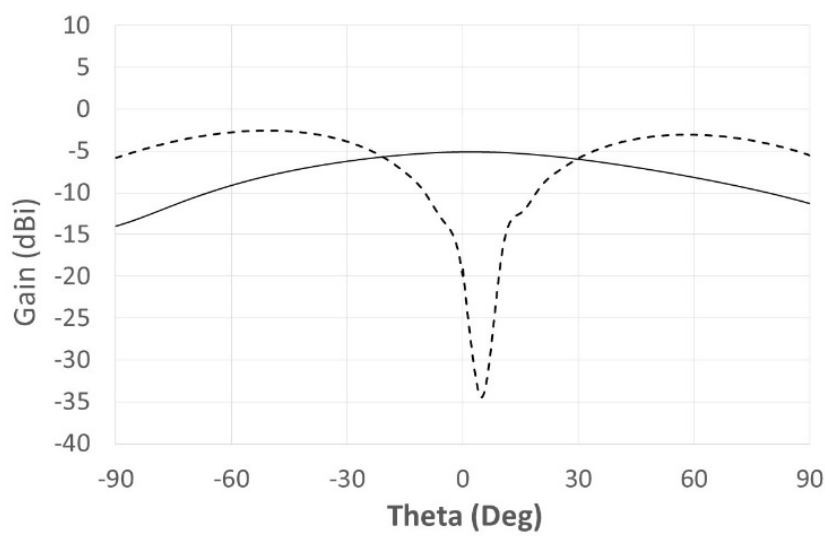

(b) 


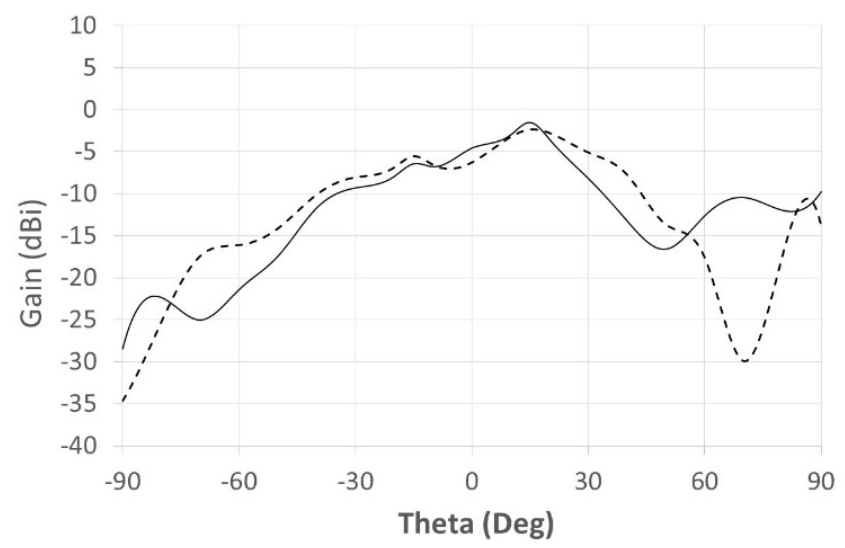

(c)

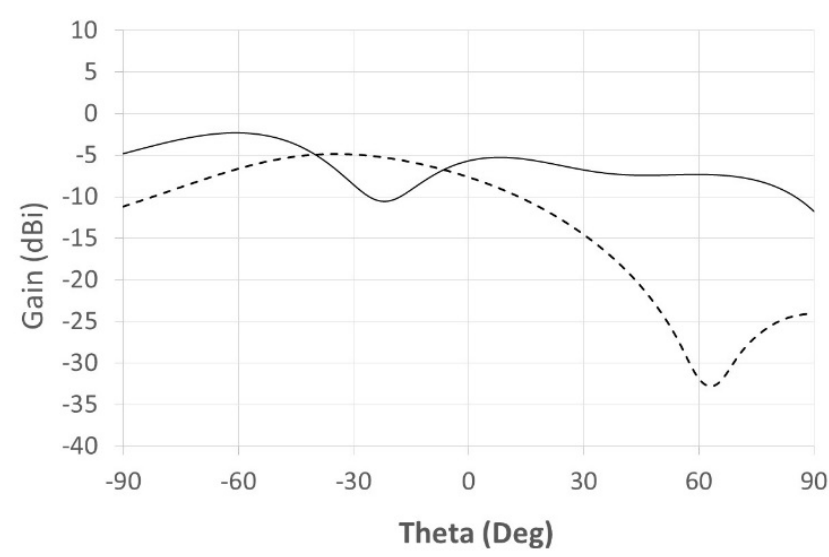

(d)

Fig.9 (a) Measured and (b) Modelled results showing the radiation pattern changes for the TM010 mode. (c) Measured (d) Simulated results for $\mathrm{TM}_{200}$ at 20GHz. dashed : without illumination, solid : with illumination

The effect of the losses on the $\mathrm{TM}_{010}$ mode due to the finite conductivity of the plasma are shown in Fig. 10a for low optical intensities and Fig. 10b for medium to high optical intensities. Results for the other modes were found to follow a similar trend. The initial dip in gain in Fig 10a is due to the heavy losses in the plasma at the lower conductivity values. The fields at the surface of the slot are able to penetrate further into the upper layers of the plasma thus increasing the losses. The gain starts to rise as the conductivity increases and the fields are pushed out of the plasma region such that much of the interaction takes place at the surface of the plasma where the conductivity is maximum. By increasing the illumination power to around $20 \mathrm{~W} / \mathrm{cm}^{2}$ as shown in Fig $10 \mathrm{~b}$ only a $3 \mathrm{~dB}$ penalty in gain is induced. Comparisons with simulation showed the equivalent conductivity of the plasma at this level of intensity was 200S/m.

Several methods for improving the gain were investigated. All measurements presented until now were with the 200nm ITO ground just below the fused silica as shown in Fig. 1b. By swapping these two layers around such that the ITO layer sits just above the brass ground plane with the $100 \mu \mathrm{m}$ glass layer now sitting just below the fused silica substrate it was found that the gain could be increased. Fig. 10c shows the improvement in gain due to the layer reversal. This shows an average increase of around $6 \mathrm{~dB}$ in gain. The ITO layer thickness used for our experiments was around 200nm. Further improvements in gain could be obtained by using thicker layers of ITO. 


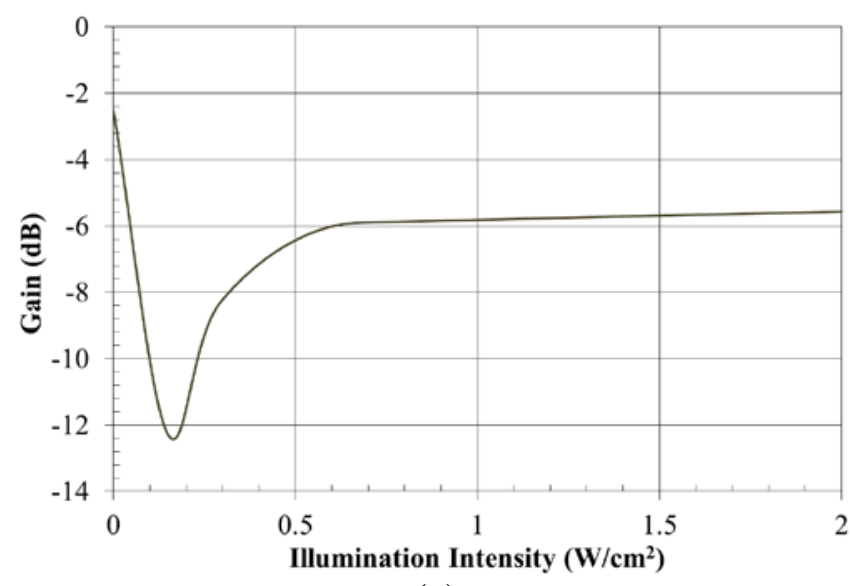

(a)

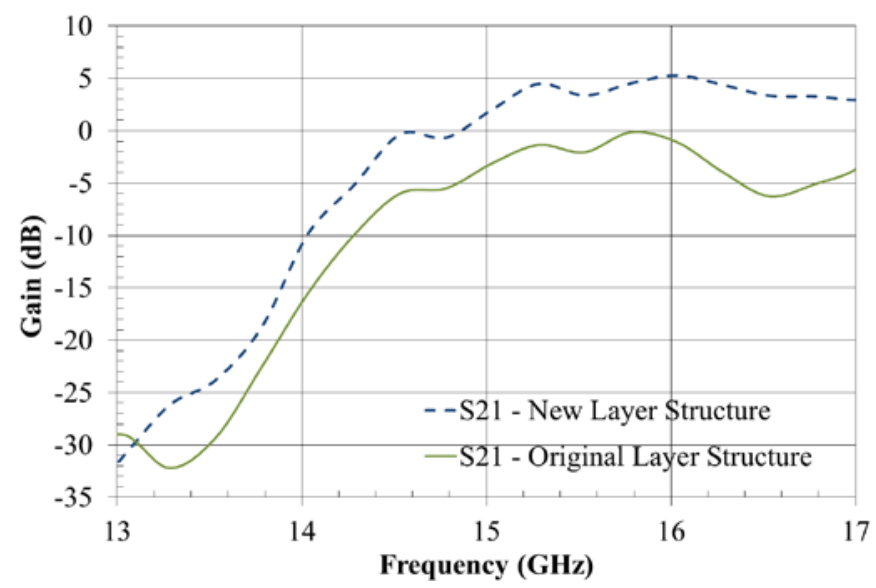

(c)

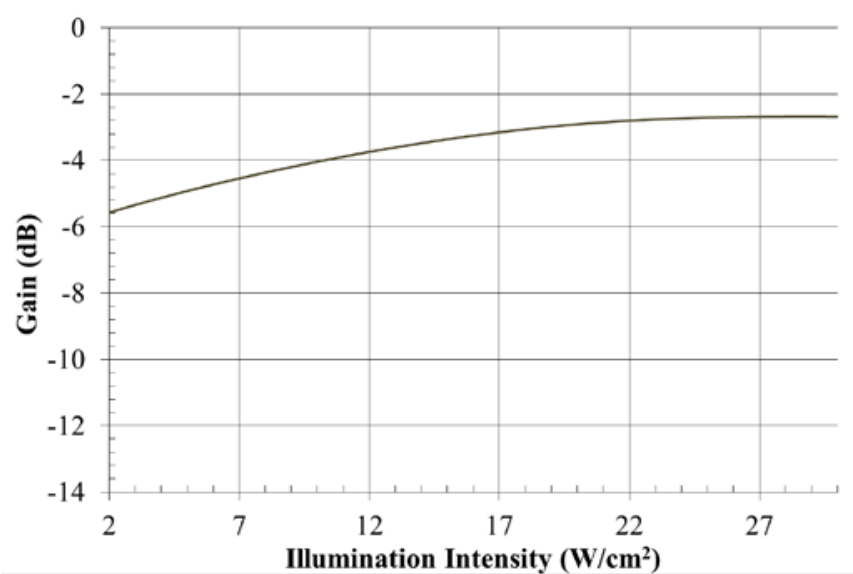

(b)

Fig. 10 Measured gain for TM010 mode (a) vs low illumination power (b) vs high illumination power for (c) with ITO layer reversed layer structure.

\section{Conclusions}

The proposed optically tunable antenna structure was investigated and simulation and measurement results presented. The results show that significant changes to the radiation patterns can be obtained using this method. Several methods for the reduction of losses were also described. The basic structure described here can be extended for the tuning of antennas at higher frequencies where more conventional switch based methods are not applicable. It was shown that this method can tune certain modes of the antenna without affecting other modes which can be an advantage for dual band antennas. The requirement for DC power for the light source currently restricts the potential applications of optical tuning technology but microLED array technology is continually increasing in its power capabilities and combined with better focusing techniques should provide the required illumination intensities at lower DC power consumption levels. 


\section{ACKNOWLEDGEMENTS}

The authors would also like to extend special thanks to Dr. Maciej Klemm for help with running the CST simulations to Dr. Andrew Murray for help with fabricating the samples and to Michael Penney for constructing the test fixtures. The Authors would like to acknowledge the Engineering and Physical Sciences Research Council (EPSRC) for funding.

Data for this paper is available from DOI : XXX

\section{References}

[1] Christos G. Christodoulou et al, "Reconfigurable Antennas for Wireless and Space Applications”, Proceedings of the IEEE, Vol. 100, No. 7, July 2012

[2] S. Lucyszyn (Ed.), “Advanced RF MEMS”, Cambridge University Press, 2010.

[3] Butler, J.K., Tran-Fu, W., and Scott, M.W., "Nonuniform layer model of a millimeter-wave phase shifter,” IEEE Trans. Microw. Theory Tech., vol. 34, no. 1, pp. 147-155, Jan. 1986.

[4] Kowalczuk, E.K., Panagamuwa, C.J., Seager R.D., Vardaxoglou Y.C., Characterising the linearity of an optically controlled photoconductive microwave switch. Loughborough Antennas and Propagation Conference, pp597600, Nov 2010.

[5] Auston, D. H., Cheung, K. P., \& Smith, P. R. Picosecond photoconducting Hertzian dipoles. Applied Physics Letters, 45(3), 284, Jan 1984.

[6] Lee, C., Mak, P., \& DeFonzo, A. Optical control of millimeter-wave propagation in dielectric waveguides. Quantum Electronics, IEEE, 16(03), 277-288. Jan 1980.

[7] Dawson, M.D., Neil, M.A.A. Micro-pixellated LEDs for science and instrumentation, Journal of Applied Physics, vol. 41, issue 9, May 2008.

[8] Edwards, R. N., Nunnally, W. C., Dickson, W. D., Miller, B. C., \& Robinette, L. K. Investigation of photoconductive silicon as a reconfigurable antenna. North American Conference on Smart Structures and Materials (pp. 344-353), Jan 1993.

[9] Liu, D., Charette, D., Bergeron, M., Karwacki, H., Adams, S., Lanning, B., \& Kustas, F. Structurally embedded photoconductive silicon bowtie antenna. IEEE Photonics Technology Letters, 10(5), 716-718, Jan 1998.

[10] C. D. Gamlath, M.A. Collett and M.J. Cryan, "Investigation of Novel Architectures for Tunable Antennas based on Optically Generated Plasmas”, 45th European Microwave Conference, Paris, September 2015

[11] Tawk, Y., Albrecht, A. R., Hemmady, S., Balakrishnan, G., \& Christodoulou, C. G.. Optically Pumped Frequency Reconfigurable Antenna Design. IEEE Antennas and Wireless Propagation Letters, Vol. 9,pp. 280-283, May 2010.

[12] Panagamuwa, C. J., Chauraya, a., \& Vardaxoglou, J. C. Frequency and Beam Reconfigurable Antenna Using Photoconducting Switches. IEEE Transactions on Antennas and Propagation, 54(2), 449-454, Feb 2006.

[13] Guo, Y.X., Luk, K. F. L. Dual Band Slot Loaded Short Circuited Patch Antenna. Electronics Letters, 36(4), 289291, Mar 2004.

[14] Dissanayake, T., \& Esselle, K. Prediction of the notch frequency of slot loaded printed UWB antennas. Antennas and Propagation, IEEE., 55(11), 3320-3325, Jan 2007.

[15] Maci, S., \& Gentili, G. B. Dual-band slot-loaded patch antenna. Antennas and Propagation, IEEE., 225-232, Mar 1995.

[16] Lee, J., Horng, T., \& Alexopoulos, N. Analysis of cavity-backed aperture antennas with a dielectric overlay. Antennas and Propagation, 42(11), 1556-1562, May 1994.

[17] Jackson, D., \& Alexopoulos, N. Gain enhancement methods for printed circuit antennas. IEEE Transactions on Antennas and Propagation, 33(9), 976-987, Jan 1985. 
[18] Carver, K., \& Mink, J. Microstrip antenna technology. IEEE Transactions on Antennas and Propagation, 29(1), 2-24, Mar 1981.

[19] Adams, A. Flush mounted rectangular cavity slot antennas--Theory and design. IEEE Transactions on Antennas and Propagation, 15(3), 342-351, Mar 1967.

[20] Gamlath, CD, Benton DM, Cryan MJ. Microwave Properties of an Inhomogeneous Optically Illuminated Plasma in a Microstrip Gap. IEEE Microwave Theory and Techniques, 63(2), 374-383. Feb 2015.

[21] Kerr, A. R. Surface impedance of superconductors and normal conductors in EM simulators. MMA Memo 21 May 1999.

[22] Collin, R. Foundations for microwave engineering. McGraw-Hill, 2001. 